communication and collaboration. For example, if the top five investors who come to mind for syndicating a deal are all from the same demographic, this should be a reminder to proactively seek other investors. Similarly, our networks influence whom we choose to fill board seats, whom we hire as $\mathrm{C}$-suite management, and the vendors we contract for drug development. If your network favors white males, that will be reflected across boards, management teams and vendors.

Venture also leans on annual offsites and elite retreats, such as a winter ski or summer golf trip, to build relationships. I would like to see more firms allocate a specific percentage of attendance to racial minorities or include a panel discussion on diversity at their offsite events. This would show a tangible commitment to being inclusive.

4. Engage in mentorship and sponsorship. I was privileged to have tremendous mentors early on in my career, many of whom were white men: my PhD advisor Larry Steinman at Stanford, James Sabry and Tom Zioncheck at Genentech Business Development, and trailblazers like Alex Schuth who formed cutting-edge companies like Denali. I attribute much of where I am today to their influence.

Part of the privilege of being a seasoned investor is paying forward your experience. That includes mentoring those who do not look like you. This requires a commitment to opening doors, allocating time to check in and be a sounding board, or simply serving as a reference. If you cannot do that, consider officially installing at your firm a mentorship program designed for young minorities. In a perfect world, you would do both.

5. Address education and culture. The death of George Floyd has initiated a dialogue where, perhaps for the first time, discussions are happening at a firm-wide level on race, including the \#blacklivesmatter movement. Although it's a positive step, for institutional change to happen these discussions need to become integrated into the overall long-term strategy, values and goals set by venture firms. As investors, we push our companies to be transparent and to set tangible, measurable milestones at each round of financing. I would like to see a matched commitment from venture firms to set similar diversity goals in the makeup of their partnerships and portfolio companies, and to be transparent about it.

It is also critical to create a culture that supports diverse backgrounds. This starts with educating ourselves on systemic racism and systems of power and privilege. Individuals with the best intentions are still working within systems that are not designed to be inclusive. Self-education is important for understanding how institutional racism works. Only when firms are educated can they put in place structures and a language with which to discuss the subconscious biases that play out in real time. Recommended reading includes White Fragility: Why It's So Hard for White People to Talk about Racism by Robin DiAngelo, When Affirmative Action Was White: An Untold History of Racial Inequality in Twentieth-Century America by Ira Katznelson and Redefining Realness by Janet Mock.

\section{Call to action}

I have hesitated to write and speak publicly on this issue because I feared I would be judged or discounted as not yet a seasoned investor or that I would alienate my white colleagues. But I am compelled to add my perspective because change is desperately needed, particularly in biotech VC. I was driven to become a biotech investor because of its ability to influence innovation. We are a regenerative force in this industry, responsible for funding the best ideas translational research brings forth. Given this power, we are particularly well poised to influence biotech diversity across several critical touchpoints, and it is imperative that we apply this notion of personal responsibility to create change in our venture community.

So while the world is navigating a confluence of events that highlight the racial inequalities that are still present today, I am reminded of the dues my father paid so that I would have opportunities that were not realized in his lifetime. That is progress. But it is not enough. How we choose to respond to this moment and the actions we take will reveal the content of our character beyond the color of our skin.

\section{Jackie Grant $₫$ \\ Abingworth, Boston, MA, USA.

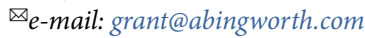

Published online: 22 July 2020

https://doi.org/10.1038/s41587-020-0624-y

\section{Competing interests}

The author declares no competing interests.

Supplementary information is available for this paper at https://doi.org/10.1038/s41587-020-0624-y.

\title{
Scalable and robust SARS-CoV-2 testing in an academic center
}

To the Editor - The emergence of the severe acute respiratory syndrome coronavirus 2 (SARS-CoV-2) (1-3 $^{1-3}$ has led to a pandemic infecting more than seven million people worldwide in less than six months, posing a major threat to healthcare systems. This is compounded by the shortage of available tests, resulting in limited staff testing programs, causing numerous healthcare workers to unnecessarily self-isolate. Here, we provide a roadmap to show how a European research institute can be repurposed in the midst of this crisis, in collaboration with partner hospitals and an established diagnostic laboratory, harnessing existing expertise in virus handling, robotics, molecular testing and informatics to derive a rapid, high-throughput diagnostic testing pipeline for detecting SARS-CoV-2 in individuals with suspected COVID-19.

Comprehensive and reliable testing is essential to identify the virus in individuals presenting with and without COVID-19 symptoms in hospital, to guide community interventions that contain the spread, and to perform enhanced surveillance of healthcare workers to maintain a workforce to safely deliver care. These requirements have placed an unprecedented demand on the testing capability of all countries. This demand on diagnostic laboratories, coupled with a global shortage of commercial kits and reagents, reduced commercial flights and cargo capacity, and international competition for testing resource, has 
rendered the testing capacity of many countries inadequate to deal with the outbreak effectively.

The pipeline we created is used to detect SARS-CoV-2 from combined nose-throat swabs and endotracheal secretions or bronchoalveolar lavage fluid. Notably, it relies on a series of in-house buffers for viral inactivation and the extraction of viral RNA, thereby reducing the dependency on commercial suppliers at times of global shortage. We use a commercial reverse transcription-PCR (RT-PCR) assay, from Shenzhen-headquartered BGI, and report the results using a custom online web application that integrates with the national healthcare digital system. This strategy allows the remote reporting of thousands of samples a day in around 24 hours, universally applicable to laboratories worldwide.

The Francis Crick Institute (the Crick) is a biomedical research institute dedicated to the discovery of biology underlying human health. Situated in central London, an epicenter of the UK pandemic, the Crick elected to repurpose its scientific and technical resources to support the immediate healthcare needs of its partner hospital, University College London Hospitals National Health Service Foundation Trust (UCLH), during the outbreak. Providing an end-to-end pipeline for clinical diagnostic testing of COVID-19, it would increase testing capacity to meet local demand and allow new surveillance programs for healthcare workers to be implemented.

Key to finding a solution was the partnership created between the Crick; UCLH, a major London healthcare provider with clinical virology expertise; and Health Services Laboratories (HSL), a UK Accreditation Service (UKAS)-recognized clinical diagnostic laboratory. Together these comprise the Crick COVID-19 Consortium (CCC). This partnership effectively removed the barriers to clinical translation and facilitated rapid implementation of robust end-to-end testing within ten days under the oversight of an accredited laboratory. Importantly, it also allowed resources and expertise to be mobilized to meet local healthcare needs (Supplementary Table 1).

A notable strength of the CCC pipeline is that it allows the testing of a wide variety of swabs that can be either dry or in any proprietary virus transport medium. These are taken at hospital sites or local drive-through stations and submitted to HSL before being transferred to the Crick. Upon arrival, specimens are barcode tracked, then proceed immediately to viral inactivation, automated extraction of viral RNA, and RT-PCR to quantify SARS-CoV-2 RNA.
Results are accessed through a custom-made online web portal, facilitating the remote analysis of data by a panel of trained reporters, and are returned to the reference laboratory. The speed and precision of the pipeline permits the reporting of thousands of samples a day, adopts processes that are widely used by many research laboratories worldwide, and is free from dependence on supply-chain constraints.

Given the urgent, two-week timeframe set to implement SARS-CoV-2 testing, it was not possible to secure clinical laboratory accreditation for the Crick to an appropriate standard, International Organization for Standardization (ISO) 15189:2012, and the equivalent College of American Pathologists (CAP) and Clinical Laboratory Improvement Amendments (CLIA) accreditation. As an alternative, the Crick took steps to ensure that the CCC test was evaluated, verified and performed for diagnostic use in an environment that adhered to equivalent international standards.

The Crick is partnering with HSL to provide diagnostic PCR testing to UCLH and other National Health Service (NHS) Trust customers of HSL. All HSL services are compliant with the UK Human Tissue Authority (HTA) and UK Medicines and Healthcare Products Regulatory Agency (MHRA) regulatory requirements, where appropriate. The Crick worked with HSL - which already had a clinically validated COVID-19 RT-PCR test against the SARS-CoV-2 nucleocapsid (N) gene - to ensure that the research institute's RT-PCR test against the SARS-CoV-2 ORF1a gene was properly audited and validated. Samples have been regularly exchanged with HSL, and the Crick laboratory has tested an external panel of samples from the UK National External Quality Assessment Service (UK NEQAS) and confirmed that the results are in accordance with UK NEQAS. The Crick laboratory is also undertaking environmental surveillance of pipeline areas.

Advice and oversight was also sought from registered professionals from existing nearby UKAS-accredited medical laboratories: HSL (UKAS 10204); Royal Marsden Hospital and North Thames Genomic Laboratory Hub (UKAS 9839); Great Ormond Street Hospital, North East Thames Regional Genetics Lab and North Thames Genomic Lab Hub (UKAS 7883); Institute of Neurology (UKAS 8045); and an approved UKAS inspector. CCC protocols were either written on demand or based on existing institutional protocols to ensure clinical-grade testing at the Crick. Guidance from these professionals assisted the compiling of clinical diagnostic standard operating procedures (SOP) for every stage of the pipeline, including implementing checklists and risk-mitigation steps alongside the methods. Additional SOPs were followed for sample storage, disposal of materials, batch certification of reagents and incident reporting. Appropriate risk assessments, training and competency assessment procedures were established and documented. Record sheets were created to document the receipt, batch acceptance testing, and start- and end-of-use dates for key reagents and consumables. An inventory of all key equipment was compiled that, where appropriate, included details of service and calibration records. Systems were established for the control of all key documents (version implementation, distribution and acknowledgement), audit trail (what samples were tested when, by whom, with what equipment and using which consumable or reagent batches), and a record of all incidents and issues (to facilitate appropriate investigation, rectification and recurrence prevention).

Assurance of the pipeline was performed in collaboration with quality assessment provider Genomic Quality Assessment (GenQA; https://www.genqa.org/), following their checklist for non-accredited laboratories. The lab and CCC workflow were inspected by a qualified UKAS assessor against the GenQA guidelines to verify compliance to IS015189 equivalent standard.

To ensure full traceability, samples were barcoded and all processes were recorded using Clarity LIMS software (Illumina). UCLH provided access to barcoded swabs prebooked onto the Crick's laboratory information management system (LIMS) to enable tracking from sample receipt through to result reporting. In urgent response to the clinical need, formation of these partnerships was vital to driving the speed of pipeline setup in the Crick's central London research laboratory. All key documents are available at https://www.crick.ac.uk/ research/covid-19/covid19-consortium.

The CCC pipeline is illustrated in Fig. 1. The specific reagents and requirements for each step of the entire pipeline-sample receipt, virus inactivation, RNA extraction, RT-PCR assay for the ORF1a gene, data quality assessment, online web reporting, barcode sample tracking - are available at protocols.io ${ }^{4}$.

In response to potential shortages of supplies, we forecast demand, ordered reagents in large batches, used in-house buffers wherever possible, and established an in-house $N$ gene assay as a contingency plan.

Several amendments to procedures were implemented to ensure the CCC test performed robustly at the Crick. Although 
a

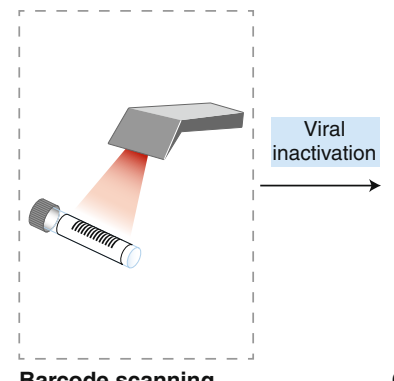

Barcode scanning

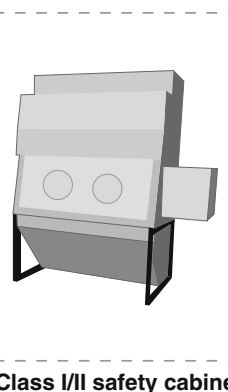

Class I/II safety cabinet

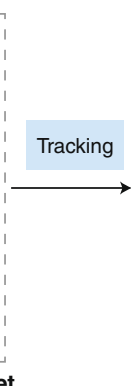

96-well plate

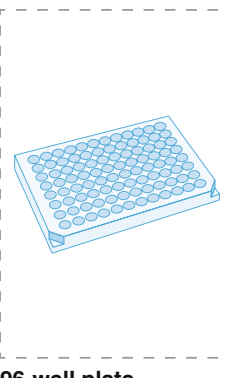

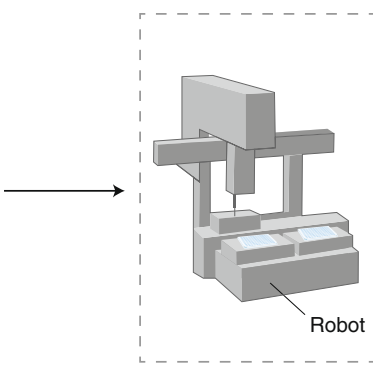

RNA extraction

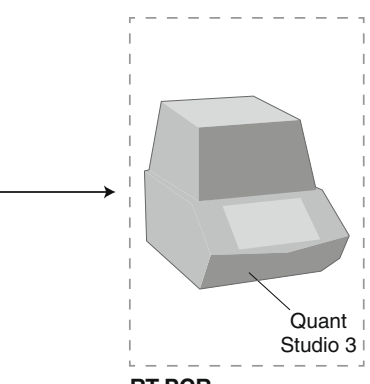

RT-PCR

b

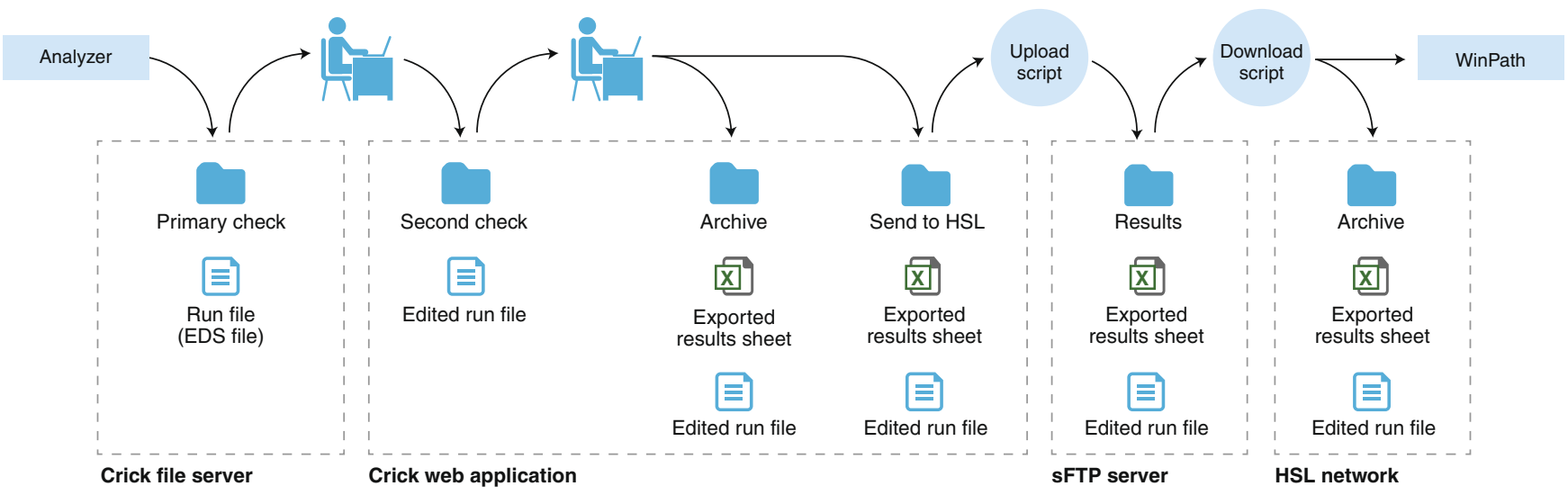

Fig. 1 | Schematic of the CCC test and reporting pipelines. a, Specimen barcodes are scanned at sample reception, before viral inactivation in a class I or II safety cabinet, processing through RNA extraction using an in-house protocol and RT-PCR testing using a commercial kit (BGI). The number of samples processed through the pipeline per day has ranged from 39 (1 plate) to 1,386 (15 plates). b, CCC reporting pipeline. Test results are reported continuously through a custom-made remote web application, allowing remote clinical scientists and pathologists working outside the institute to authorize reports, in line with the established SOP. EDS, experiment document single; sFTP, secure file transfer protocol.

the Crick performed viral inactivation in a Containment Level 3 suite with trained staff, other research laboratories with only Containment Level 2 facilities may be able to adapt the CCC test model, provided that appropriate risk assessments are carried out or swabs are inactivated before transport. A Containment Level 2 procedure is also provided with our protocols. Moreover, other protocols also exist for alternative viral-inactivation methods using heat, further demonstrating the potential for CCC pipeline applicability where availability of guanidinium may be limited.

Another adaptation made in the CCC test is to use a series of homemade buffers for automated RNA extraction, which circumvents dependence on reagents that may be in short supply during a pandemic. At the time of writing, the most important bottleneck in performing PCR tests for COVID-19 detection is the shortage of kits for RNA extraction. We developed an in-house RNA extraction protocol using magnetic silica beads from G-Biosciences, and we have also validated our assay with SeraSil Mag 400 beads (GE Healthcare/ Cytiva), which can serve as a reliable substitute. RNA extraction using silica beads is based on the protocol developed by Boom et al. ${ }^{5}$ over 30 years ago. In the Boom method, concentrated guanidinium thiocyanate serves as virus and RNase inactivation agent and promotes binding of nucleic acids to silica. We have tested RNAclean XP SPRI magnetic beads (Beckman) and found them compatible with our virus inactivation solution; viral RNA could be purified following manufacturer's recommendations. Moreover, protocols exist for the production of either type of magnetic beads from inexpensive and accessible starting materials ${ }^{6}$. Therefore, we designed a pipeline that uses common reagents and is automatable on widely available liquid-handling platforms, allowing its implementation in a large number of biomedical laboratories with suitable robotic platforms that can be reprogrammed for this use. The reagents can also be used for manual RNA extraction where liquid-handling platforms are unavailable. The universal applicability of this approach could allow a resilient response to future critical events, even in countries where particular resources may be limited.

Selection of an appropriate PCR assay for detection of SARS-CoV-2, the
BGI kit, was based on (i) our accredited laboratory having a ready set of validation data and experience with the assay, which has a US Food and Drug Administration Emergency Use Authorization, and (ii) a guaranteed supply chain for the assay kit in the face of falling demand in China and growing demand in the United States (for US suppliers). The primers used in the CCC test target SARS-CoV-2 ORF1a, enabling detection of full-length genomic and antigenomic RNA, whereas the $N$ gene assay also targets the abundant subgenomic RNAs. With many mutations having been reported in the ORF1 region of SARS-CoV-2, it was paramount to adequately assess false-positive and false-negative rates. The verification steps of the CCC pipeline allowed us to compare the BGI kit with the in-house developed $N$ gene assay. Overall, the diagnostic sensitivity of the CCC test is $92.86 \%$, with a specificity of $100 \%$ (based on a small sample set of specimens from patients infected with other seasonal respiratory viruses) and a high degree of accuracy in the detection of SARS-CoV-2. The $N$ gene assay is slightly more sensitive than the CCC assay at the limits of detection. When performed in 
duplicate, we observed a discordant rate of $1.1 \%$ (95 of 8,433 samples). To improve the accuracy of true positive reporting and reduce the chance of reporting false positive tests, the assay is performed in duplicate and discordant samples are recommended for retesting at the source (see "Additional Information for Scalable and Robust SARS-CoV-2 Testing in an Academic Center" on Figshare ${ }^{7}$.

As sample timing and adequacy are likely to be more important determinants of false negatives than quantitative PCR sensitivity ${ }^{8}$, we have chosen a test that also includes a control for cellular RNA ( $\beta$-actin), which serves as a partial proxy for sample adequacy. Although high sensitivity at the assays limits of detection could affect identification of low levels of viral shedding beyond the assay's limits of detection, these samples are unlikely to be producing infectious virus ${ }^{9}$. BGI PCR therefore exhibits adequate sensitivity for current clinical algorithms, in which testing for symptomatic healthcare workers is performed within a specific timeframe.

The high-throughput RT-PCR assay carried out at the Crick in 96-well plate format has the potential to screen thousands of samples per day and can be scaled up to 384-well format with further optimization. Since inception of the CCC, we have performed over 30,000 tests as of 12 June 2020 , starting with one batch of 39 samples on the first day of live testing and scaling up to around 500-1,000 samples per day. The turnover of 1,000 samples/day is delivered on a pipeline operated by 44 members of Crick scientific staff working a 10-h (staggered) shift. Competency training was conducted for staff to work on virus inactivation, RNA extraction, RT-PCR and result reporting. This is far less than our maximum capacity for CCC testing, and throughput is limited by logistic and operational considerations within the community and partner hospitals (for example, local swabbing capacity) and national guidelines. Our partner laboratory HSL has capacity for 1,000-1,250 samples per day, but owing to limitations on supply chain, their testing is reserved for hospital inpatients. By contrast, the CCC pipeline created capacity to test a new population that had hitherto been unable to access testing: asymptomatic and symptomatic healthcare workers and self-isolating key workers. Indeed, we now provide the healthcare worker testing for UCLH NHS Trust and North Central London Hospitals. We believe that this approach fulfilled a critical gap in the existing testing infrastructure, and one that has a major impact on the safe delivery of healthcare during the COVID-19 pandemic.
To establish the CCC pipeline at the Crick, we took Containment Level 3 equipment, liquid-handling platforms and RT-PCR instruments that were available in our institute and repurposed them for COVID-19 testing. The barcoding equipment and tool tracker were already used with our LIMS system. Only a limited amount of extra protective equipment was procured for buffer preparation, and the pipeline can be potentially scaled up further with minimal extra equipment. A rate-limiting step preventing the CCC pipeline from proceeding at full capacity is the global availability of swabs. Additional testing regimens are being considered that would circumvent the dependency on viral swabs.

Medical laboratory accreditation is held to the standard of ISO 15189:2012 across the world, with the exception of the United States, which operates to CLIA certification and CAP accreditation. Laboratories are assessed for compliance to ISO or CLIA/CAP standard by a national awarding body; in the case of the United Kingdom this body is UKAS. Although the process of acquiring accreditation and the typical assessment time span and rules for extending existing ISO or CLIA/CAP scope to partnering institutions will vary between countries, any research institution seeking to establish clinical testing should seek clinical accreditation wherever possible. While pursuing this process, our approach has been to implement processes in line with international accreditation standards, and those processes remain under the supervision of our partner accredited laboratory (HSL). We have also regularly sought advice from GenQA and are in the process of implementing their recommendations in an agreed timeframe to comply with the standards required to meet ISO15189.

Health information systems, such as the EPIC electronic medical records used at UCLH, interface with LIMS, such as WinPath, to enable sample barcodes to be associated with patient hospital numbers. The pipeline set up at the Crick uses a custom-made reporting web application compatible with remote reporting. This allows multiple trained reporters to access anonymized data through a portal from home, which is particularly advantageous in a pandemic. As a result, the CCC pipeline is capable of an accelerated turnaround for results of 2,500-3,000 samples in approximately $24 \mathrm{~h}$.

The potential advantages of implementing a clinical diagnostic pipeline in research laboratories are clear: a substantial increase in capacity for testing and the ability to adopt flexible and agile approaches to testing in the face of global constraints. Our experience at the Crick in implementing mass-scale testing in the CCC has taught us invaluable lessons for the wider academic community: first, diagnostic testing to clinical standards can be successfully achieved through partnership and guidance from a clinical diagnostic laboratory; second, the choice of techniques and approaches should be adapted to local resources and staff expertise, already existing within a research laboratory; and third, the scale and implementation of testing should be aligned with the healthcare needs and demands of the local population.

\section{Editorial note: This article has been peer reviewed.}

Jim Aitken', Karen Ambrose', Sam Barrell', Rupert Beale ${ }^{1,2}$, Ganka Bineva-Todd', Dhruva Biswas², Richard Byrne', Simon Caidan', Peter Cherepanov', Laura Churchward ${ }^{3}$, Graham Clark', Margaret Crawford', Laura Cubitt', Vicky Dearing', Christopher Earl', Amelia Edwards', Chris Ekin ${ }^{4}$, Efthymios Fidanis',

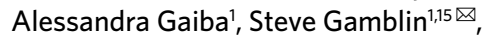
Sonia Gandhi',2,3,15凶 , Jacki Goldman', Robert Goldstone', Paul R. Grant ${ }^{4,14}$, Maria Greco', Judith Heaney ${ }^{3}$, Steve Hindmarsh', Catherine F. Houlihan ${ }^{3}$, Michael Howell', Michael Hubank ${ }^{5,6}$, Deborah Hughes ${ }^{5}$, Rachael Instrell', Deborah Jackson', Mariam Jamal-Hanjani ${ }^{2,3}$, Ming Jiang', Mark Johnson', Leigh Jones' ${ }^{1}$ Nnennaya Kanu 2,14, George Kassiotis', Stuart Kirk ${ }^{4}$, Svend Kjaer', Andrew Levett ${ }^{4}$, Lisa Levett ${ }^{4}$, Marcel Levi ${ }^{3}$, Wei-Ting Lu', James I. MacRae', John Matthews ${ }^{4}$, Laura E. McCoy ${ }^{2}$, Catherine Moore David Moore ${ }^{2,3}$, Eleni Nastouli3,8,15凶, Jerome Nicod', Luke Nightingale', Jessica Olsen', Nicola O'Reilly (D1, Amar Pabari ${ }^{4}$, Venizelos Papayannopoulos', Namita Patel', Nigel Peat', Marc Pollitt', Peter Ratcliffe', Caetano Reis e Sousa', Annachiara Rosa', Rachel Rosenthal', Chloe Roustan', Andrew Rowan', Gee Yen Shin ${ }^{3,4}$, Daniel M. Snell', Ok-Ryul Song', Moira J. Spyer', Amy Strange 1, Charles Swanton 1,2,3,15凶, James M. A. Turner', Melanie Turner", AndreasWack', PhilipA. Walker', Sophia Ward ${ }^{1,2}$, Wai Keong Wong ${ }^{3}$, Joshua Wright (D)', Mary Wu' and The Crick COVID-19 Consortium*

${ }^{1}$ The Francis Crick Institute, London, UK. ${ }^{2}$ University College London, London, UK. ${ }^{3}$ University College London Hospitals, NHS Foundation Trust, London, UK. ${ }^{4}$ Health Services Laboratories, London, UK. ${ }^{5}$ The Institute of Cancer Research, London, UK. ${ }^{6}$ The Royal Marsden Hospital, Surrey, UK. ${ }^{7}$ Public Health Wales, Heath Park, Cardiff, UK. ${ }^{8}$ University College London GOS Institute of Child Health, London, UK. ${ }^{14}$ These authors contributed equally: Paul R. Grant, 
Nnennaya Kanu. ${ }^{15}$ These authors jointly directed this work: Steve Gamblin, Sonia Gandhi, Eleni Nastouli, Charles Swanton. ${ }^{*} A$ list of authors and their affiliations appears at the end of the paper.

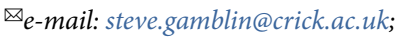

sonia.gandhi@crick.ac.uk; eleni.nastouli@nhs.net; Charles.Swanton@crick.ac.uk

Published online: 18 June 2020

https://doi.org/10.1038/s41587-020-0588-y

References

1. Jiang, S., Du, L. \& Shi, Z. Emerg. Microbes Infect. 9, 275-277 (2020).

2. Zhou, P. et al. Nature $579,270-273(2020)$.

3. Zhu, N. et al. N. Engl. J. Med. 382, 727-733 (2020).

4. Crick COVID-19 Consortium. The Crick COVID-19 RT-PCR testing pipeline. protocols.io https://doi.org/10.17504/protocols. io.bfe2ijge (2020).

5. Boom, R. et al. J. Clin. Microbiol. 28, 495-503 (1990).

6. Oberacker, P. et al. PLoS Biol. 17, e3000107 (2019).

7. Crick COVID-19 Consortium. Additional information for "Scalable and robust SARS-CoV-2 testing in an academic center". Figshare https://hdl.handle.net/10779/crick.c.4976444 (2020).

8. Wikramaratna, P. et al. Estimating false-negative detection rate of SARS-CoV- 2 by RT-PCR. Preprint at medRxiv https://doi. org/10.1101/2020.04.05.20053355 (2020).

9. Wölfel, R. et al. Nature 581, 465-469 (2020)

\section{Acknowledgements}

The authors wish to thank Heather Ringrose for support with the Hamilton liquid handling workstation. This work was supported by the Francis Crick Institute, which receives its core funding from Cancer Research UK (FC001169, FC001078), the UK Medical Research Council (FC001169, FC001078) and the Wellcome Trust (FC001169, FC001078). C.S. is Royal Society Napier Research Professor and is also funded by the Breast Cancer Research Foundation. E.N. receives research funding from the NIHR, MRC, GSK and H2020. S. Gandhi is an MRC Senior Clinical Fellow.

\section{Competing interests}

C.S. receives grant support from Pfizer, AstraZeneca, Bristol-Myers Squibb (BMS), Roche-Ventana, Boehringer-Ingelheim and Ono Pharmaceutical and has consulted or received an honorarium from Pfizer, Novartis, GlaxoSmithKline, Merck Sharp \& Dohme, BMS, Celgene, AstraZeneca, Illumina, Genentech, Roche-Ventana, GRAIL, Medicxi and the Sarah Cannon Research Institute. C.S. is a shareholder of Apogen Biotechnologies, Epic Bioscience and GRAIL and has stock options in and is a cofounder of Achilles Therapeutics. M. Hubank has received laboratory support, consultancy fees or advisory board membership from Guardant Health, Roche Diagnostics, Boehringer-Ingelheim, BMS and AstraZeneca.

\section{Additional information}

Supplementary information is available for this paper at https://doi.org/10.1038/s41587-020-0588-y.

The Crick COVID-19 Consortium

Jim Aitken', Zoe Allen', Rachel Ambler', Karen Ambrose', Emma Ashton ${ }^{9}$, Alida Avola', Samutheswari Balakrishnan',

Caitlin Barns-Jenkins', Genevieve Barr',

Sam Barrell', Souradeep Basu', Rupert Beale 1,2, Clare Beesley ${ }^{9}$, Nisha Bhardwaj', Shahnaz Bibi ${ }^{9}$, Ganka Bineva-Todd', Dhruva Biswas², Michael J. Blackman ${ }^{1,10}$, Dominique Bonnet ${ }^{1}$, Faye Bowker', Malgorzata Broncel', Claire Brooks ${ }^{9}$, Michael D. Buck',
Andrew Buckton ${ }^{9}$, Timothy Budd', Alana Burrell', Louise Busby ${ }^{9}$, Claudio Bussi', Simon Butterworth', Fiona Byrne', Richard Byrne', Simon Caidan', Joanna Campbell"1, Johnathan Canton', Ana Cardoso', Nick Carter', Luiz Carvalho', Raffaella Carzaniga', Natalie Chandler ${ }^{9}$, Qu Chen', Peter Cherepanov', Laura Churchward ${ }^{3}$, Graham Clark?, Bobbi Clayton', Clementina Cobolli Gigli', Zena Collins', Sally Cottrell ${ }^{9}$, Margaret Crawford', Laura Cubitt', Tom Cullup ${ }^{9}$, Heledd Davies', Patrick Davis', Dara Davison', Vicky Dearing', Solene Debaisieux', Monica Diaz-Romero', Alison Dibbs', Jessica Diring', Paul C. Driscoll', Annalisa D'Avola', Christopher Earl', Amelia Edwards 1 , Chris Ekin ${ }^{4}$, Dimitrios Evangelopoulos ${ }^{1,2}$, Rupert Faraway 1,2, Antony Fearns', Aaron Ferron', Efthymios Fidanis', Dan Fitz', James Fleming', Bruno Frederico', Alessandra Gaiba', Anthony Gait ${ }^{9}$, Steve Gamblin ${ }^{1}$ Sonia Gandhi',2,3, Liam Gaul', Helen M. Golding', Jacki Goldman', Robert Goldstone', Belen Gomez Dominguez' ${ }^{9}$, Hui Gong', Paul R. Grant ${ }^{4}$, Maria Greco', Mariana Grobler ${ }^{9}$, Anabel Guedan', Maximiliano G. Gutierrez', Fiona Hackett' ${ }^{1}$, Ross Hall', Steinar Halldorsson', Suzanne Harris' ${ }^{1}$, Sugera Hashim ${ }^{9}$, Lyn Healy ${ }^{1}$, Judith Heaney ${ }^{3}$, Susanne Herbst', Graeme Hewitt ${ }^{1}$, Theresa Higgins', Steve Hindmarsh', Rajnika Hirani', Joshua Hope', Elizabeth Horton', Beth Hoskins ${ }^{9}$, Catherine F. Houlihan ${ }^{3}$, Michael Howell', Louise Howitt', Jacqueline Hoyle', Mint R. Htun', Michael Hubank ${ }^{5,6}$, Hector Huerga Encabo', Deborah Hughes ${ }^{5}$, Jane Hughes', Almaz Huseynova', Ming-Shih Hwang', Rachael Instrell', Deborah Jackson', Mariam Jamal-Hanjani ${ }^{2,3}$, Lucy Jenkins ${ }^{9}$, Ming Jiang', Mark Johnson', Leigh Jones ${ }^{1}$, Nnennaya Kanu' ${ }^{2}$, George Kassiotis', Louise Kiely ${ }^{9}$, Anastacio King Spert Teixeira ${ }^{1}$ Stuart Kirk', Svend Kjaer', Ellen Knuepfer ${ }^{1,2}$, Nikita Komarov ${ }^{1,2}$, Paul Kotzampaltiris ${ }^{11}$, Konstantinos Kousis', Tammy Krylova', Ania Kucharska1, Robyn Labrum", Catherine Lambe', Michelle Lappin', Stacey-Ann Lee ${ }^{1}$, Andrew Levett ${ }^{4}$, Lisa Levett ${ }^{4}$, Marcel Levi ${ }^{3}$, Hon Wing Liu', Sam Loughlin ${ }^{9}$, Wei-Ting Lu', James I. MacRae', Akshay Madoo', Julie A. Marczak', Mimmi Martensson', Thomas Martinez', Bishara Marzook', John Matthews ${ }^{4}$, Joachim M. Matz', Samuel McCall"1, Laura E. McCoy ${ }^{2}$, Fiona McKay ${ }^{9}$, Edel C. McNamara', Carlos M. Minutti', Gita Mistry1', Miriam Molina-Arcas', Beatriz Montaner', Kylie Montgomery' Catherine Moore ${ }^{7}$, David Moore ${ }^{2,3}$, Anastasia Moraiti', Lucia Moreira-Teixeira',
Joyita Mukherjee',

Cristina Naceur-Lombardelli², Eleni Nastouli ${ }^{3,8}$, Aileen Nelson', Jerome Nicod', Luke Nightingale', Stephanie Nofal', Paul Nurse', Savita Nutan', Caroline Oedekoven', Anne O'Garra', Jean D. O'Leary', Jessica Olsen', Olga O'Neill', Nicola O'Reilly', Paula Ordonez Suarez', Neil Osborne', Amar Pabari ${ }^{4}$,

Aleksandra Pajak',

Venizelos Papayannopoulos', Namita Patel', Yogen Patel"1, Oana Paun', Nigel Peat', Laura Peces-Barba Castano', Ana Perez Caballero ${ }^{9}$, Jimena Perez-Lloret ${ }^{1}$, Magali S. Perrault' ${ }^{1}$, Abigail Perrin', Roy Poh ${ }^{11}$, Enzo Z. Poirier ${ }^{1}$, James M. Polke ${ }^{11}$, Marc Pollitt ${ }^{1}$, Lucia Prieto-Godino', Alize Proust', Clinda Puvirajasinghe ${ }^{9}$, Christophe Queval', Vijaya Ramachandran ${ }^{9}$, Abhinay Ramaprasad', Peter Ratcliffe', Laura Reed ${ }^{9}$,

Caetano Reis e Sousa', Kayleigh Richardson', Sophie Ridewood', Fiona Roberts',

Rowenna Roberts ${ }^{9}$, Angela Rodgers ${ }^{1}$, Pablo Romero Clavijo', Annachiara Rosa', Rachel Rosenthal', Alice Rossi', Chloe Roustan', Andrew Rowan', Erik Sahai', Aaron Sait', Katarzyna Sala', Theo Sanderson', Pierre Santucci', Fatima Sardar', Adam Sateriale', Jill A. Saunders', Chelsea Sawyer ${ }^{1}$, Anja Schlott', Edina Schweighoffer', Sandra Segura-Bayona', Rajvee Shah Punatar', Joe Shaw ${ }^{9}$, Gee Yen Shin ${ }^{3,4}$, Mariana Silva Dos Santos', Margaux Silvestre', Matthew Singer ${ }^{1}$, Daniel M. Snell', Ok-Ryul Song', Moira J. Spyer ${ }^{2}$, Louisa Steel ${ }^{9}$, Amy Strange', Adrienne E. Sullivan', Charles Swanton ${ }^{1,2,3}$, Michele S. Y. Tan', Zoe H. Tautz-Davis', Effie Taylor', Gunes Taylor', Harriet B. Taylor' ${ }^{1}$ Alison Taylor-Beadling,

Fernanda Teixeira Subtil', Berta Terré Torras', Patrick Toolan-Kerr ${ }^{1,2}$, Francesca Torelli', Tea Toteva', Moritz Treeck', Hadija Trojer ${ }^{13}$, Ming-Han C. Tsai', James M. A. Turner', Melanie Turner ${ }^{4}$, Jernej Ule ${ }^{1,2}$, Rachel Ulferts ${ }^{1}$, Sharon P. Vanloo ${ }^{1,2}$, Selvaraju Veeriah ${ }^{2}$, Subramanian Venkatesan', Karen Vousden', Andreas Wack', Claire Walder ${ }^{9}$,

Philip A. Walker', Yiran Wang', Sophia Ward ${ }^{1,2}$, Catharina Wenman' ${ }^{9}$, Luke Williams', Matthew J. Williams', Wai Keong Wong'3, Joshua Wright', Mary Wu', Lauren Wynne', Zheng Xiang', Melvyn Yap', Julian A. Zagalak',2, Davide Zecchin ${ }^{1,8}$ and Rachel Zillwood ${ }^{1}$ ${ }^{9}$ Great Ormond Street Hospital for Sick Children NHS Foundation Trust, London, UK. ${ }^{10}$ London School of Hygiene \& Tropical Medicine, London, UK. ${ }^{11}$ National Hospital for Neurology and Neurosurgery, University College London Hospitals NHS Foundation Trust, London, UK. ${ }^{12}$ Department of Pathobiology and Population Sciences, Royal Veterinary College, University of London, London, UK. ${ }^{13}$ Royal Free London NHS Foundation Trust, London, UK. 